\title{
Soluções individuais para o sistema de esgotamento sanitário de um loteamento rural na cidade de Sertaneja-PR.
}

Individual solutions for a rural lot sewage treatment system in Sertaneja-PR

Soluciones individuales para el sistema de alcantarillado sanitario de una parcelación rural en la ciudad de Sertaneja-PR

Alyna Mayumi Michelato Ougo Discente, UNIFIL, Brasil alyna_ougo@hotmail.com

Rafael Montanhini Soares de Oliveira Professor Doutor, UTFPR, Brasil rafaeloliveira@utfpr.edu.br

Isabela Bruna de Tavares Machado Bolonhesi Professora Doutoranda, UEL, Brasil ibtmachado@gmail.com 


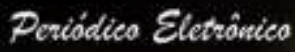

\section{RESUMO}

O saneamento na área rural é restrito e necessita de mecanismos alternativos para tratamento de esgoto com o intuito de melhorar a qualidade de vida da população. $O$ objetivo desse trabalho foi propor projetos alternativos de tratamento de esgoto para áreas rurais por meio de sistemas individuais como tanque séptico seguido de wetland (TS+W) e a fossa biodigestora (FB) modelo EMBRAPA. Os projetos propostos foram comparados com o método convencionalmente adotado para solução individual: tanque séptico seguido de filtro anaeróbio e sumidouro (TS+FA+S). A determinação do sistema convencional seguiu a metodologia da NBR 7229/1993 e NBR 13969/1997. Para o projeto de wetland, foi proposto um sistema de fluxo superficial composto por Zantedeschia Aethiopica (copos-de-leite) como macrófitas, enquanto a proposta de fossa digestora foi composta por um sistema de fluxo horizontal composto por três caixas de plástico de $1000 \mathrm{~L}$ cada. A partir do dimensionamento dos sistemas, o orçamento foi obtido por meio de pesquisas em depósitos de materiais de construção. Como resultado, concluiu-se que o sistema convencional ( $T S+F A+S)$ foi o sistema que ocupou maior área ao ser dimensionado. A fossa biodigestora é uma alternativa viável, porém não é o sistema mais barato, sendo o tanque séptico seguido de wetland um mecanismo de tratamento barato, eficiente e que promove sustentabilidade ao sistema de tratamento de esgoto, sobretudo em áreas rurais.

PALAVRAS-CHAVE: Wetland. Fossa biodigestora. Saneamento.

\section{ABSTRACT}

Rural area sanitation is restricted and requires alternative mechanisms for sewage treatment with the aim of improving the population quality of life. This work has the aim to propose an alternative sewage treatment projects for rural areas through individual systems such as septic tank followed by wetland (TS+W) and the EMBRAPA biodigester (FB). The proposed projects were compared with the conventionally method adopted for individual solution: septic tank followed by anaerobic filter and sink (TS+FA+S). The determination of the conventional system was according to NBR 7229/1993 and NBR 13969/1997. For the wetland project a surface flow system composed of Zantedeschia Aethiopica (Calla Lily) as macrophytes was proposed, while the biodigester proposed was composed of a horizontal flow system composed of three plastic boxes of $1000 \mathrm{~L}$ each. The budget was obtained through research on deposits of construction materials. As a result, it was concluded that the conventional system (TS+FA+S) had the greater area size. The biodigester was a viable alternative, but was not the cheapest system, while the septic tank followed by wetland is an inexpensive, efficient treatment mechanism that promotes sustainability to the sewage treatment system, especially in rural areas.

PALAVRAS-CHAVE: Wetland. Biodigester. Sanitation.

\section{RESUMEN}

El saneamiento en el área rural es restringido y necesita mecanismos alternativos para tratamiento de aguas residuales con el objetivo de mejorar la calidad de vida de la población. El objetivo de este trabajo fue proponer proyectos alternativos de tratamiento de aguas residuales para áreas rurales a través de sistemas individuales como tanque séptico seguido de wetland (TS $+W$ ) y la fosa biodigestora (FB) modelo EMBRAPA. Los proyectos propuestos fueron comparados con el método convencionalmente adoptado para solución individual: tanque séptico seguido de filtro anaerobio y sumidero $(T S+F A+S)$. La determinación del sistema convencional siguió la metodología de la NBR 7229/1993 y NBR 13969/1997. Para el proyecto de wetland, se propuso un sistema de flujo superficial compuesto por Zantedeschia Aethiopica (vasos de leche) como macrófitas, mientras que la propuesta de fosa digestora fue compuesta por un sistema de flujo horizontal compuesto por tres cajas de plástico de 1000 L cada uno. A partir del dimensionamiento de los sistemas, el presupuesto fue obtenido por medio de investigaciones en depósitos de materiales de construcción. Como resultado, se concluyó que el sistema convencional (TS+FA+S) fue el sistema que ocupó mayor área al ser dimensionado. La fosa biodigestora es una alternativa viable, pero no es el sistema más barato, siendo el tanque séptico seguido de wetland un mecanismo de tratamiento barato, eficiente y que promueve sostenibilidad al sistema de tratamiento de aguas residuales, sobre todo en áreas rurales.

PALAVRAS-CHAVE: Wetland. Fosa biodigestora. Saneamiento. 


\section{INTRODUÇÃO}

Estima-se que 29,9 milhões de pessoas residiam em localidades rurais no Brasil no último levantamento geográfico brasileiro, totalizando, aproximadamente, 8,1 milhões de domicílios (Instituto Brasileiro de Geografia e Estatística -IBGE, 2011). Para essas comunidades, os serviços de saneamento prestados são escassos e apresentam elevado déficit de cobertura. $O$ maior déficit ocorre para o esgotamento sanitário, em que $54 \%$ dos domicílios possuem atendimento precário, enquanto $29 \%$ são considerados sem atendimento (IBGE, 2011; Plano Nacional de Saneamento Básico - PLANSAB, 2013). Ou seja, há um grande número de pessoas que ainda habitam locais desprovidos de sistemas básicos de coleta e tratamento de esgoto.

A implantação de redes de coleta de esgotamento sanitário em áreas rurais é importante para garantir qualidade de vida aos indivíduos dessa comunidade. Uma das principais dificuldades de implantação de redes de esgoto na zona rural é a distância elevada entre as residências, o que encarece ou inviabiliza esse sistema (GONÇALVES et al. 2013).

Quando não é possível o sistema coletivo com a implantação de redes coletoras, sistemas alternativos de tratamento de esgoto podem ser implantados para minimizar impactos ambientais e sanitários. Nas áreas rurais, na maioria das residências (aproximadamente 75\%), esse material é descartado em fossas negras ou rudimentares que não possuem isolamento adequado do solo, podendo causar a contaminação de cursos d'água (SILVA et al. 2015; GONTIJO, 2016). Diante disso, o desenvolvimento de estudos que unam práticas de adequação e sustentabilidade para coleta e tratamento de esgoto é importante.

O sistema abordado no presente estudo é uma proposta de tratamento individual composto por tanque séptico seguido de wetland e a fossa biodigestora modelo Embrapa. O tanque séptico tem a finalidade de preservar a qualidade das águas superficiais e subterrâneas, não sendo possível a adição de despejos no sistema que possam causar grandes interferências na rede, como por exemplo, o acúmulo de águas pluviais (NORMAS TÉCNICAS BRASILEIRAS - NBR 7229, 1993; SPERLING, 2014). Como pós-tratamento do tanque séptico, o sistema wetlands pode ser definido, como um tratamento de águas residuárias que consiste na introdução de plantas para remoção de matéria orgânica e nutrientes e oxigenação do solo (LEMES et al. 2008).

O sistema de tratamento formado pela fossa biodigestora consiste na substituição do modelo convencional de fossa séptica seguida de pós-tratamento por meio de três caixas de plástico ou cimento amianto interligadas. Este processo efetua o tratamento por meio de digestão anaeróbica da matéria orgânica por microrganismos em três etapas, gerando biogás e biofertilizante possivelmente utilizável em destinos agrícolas (NOVAES et al., 2002). 0 biodigestor tem dois propósitos: mudar o custo acessível para o produtor rural, o esgoto desprotegido e os tanques sépticos; e aproveitar o resíduo como um fertilizante orgânico, reduzindo custos com fertilizantes químicos, isto é beneficiar o saneamento rural e melhorar a agricultura orgânica (PERES et al., 2010). 
Dessa forma, esse artigo teve como objetivo propor duas soluções individuais para o sistema de esgotamento sanitário de um loteamento rural na cidade de Sertaneja-PR e compara-las ao método convencional de solução individual (tanque séptico seguido de filtro anaeróbio e sumidouro - TS+FA+S). Portanto, são pontuados como objetivos específicos: i) Propor um projeto de tratamento de tanque séptico combinado com wetland (TS+W); ii) propor projeto de tratamento composto por fossa biodigestora (FB) modelo Embrapa; iii) Analisar a viabilidade econômica das duas soluções propostas em relação ao modelo convencional $(\mathrm{TS}+\mathrm{FA}+\mathrm{S})$.

\section{METODOLOGIA}

\subsection{Caracterização da área de estudo}

As propostas de soluções individuais de tratamento de esgoto foram elaboradas com o propósito de futuramente serem implantadas em um loteamento rural de $41.594,07 \mathrm{~m}^{2}$ localizado a $12 \mathrm{~km}$ do Município de Sertaneja - PR, próximo ao Rio Paranapanema, que deverá ter 65 lotes e construção de uma casa por lote.

\subsection{Dimensionamento das soluções individuais de esgotamento sanitário}

A proposição de métodos de tratamento sustentáveis para uma comunidade rural, considerou um alojamento provisório e taxa mínima de cinco pessoas por residência.

O sistema convencional de tratamento de esgoto para soluções individuais é formado pelo tanque séptico (TS) seguido de filtro anaeróbio (FA) e sumidouro (S), definido de acordo com as características geológicas do solo local. Para dimensionamento do tanque séptico seguiu-se a NBR 7229/1993. Como o sistema servirá um loteamento de área rural de 65 lotes, com maior ocupação em finais de semana, adotou-se como contribuição diária de esgoto (C) o valor de 80L. hab. dia ${ }^{-1}$, segundo a Tabela 1 da NBR 7229/1993. Considerando que o período de detenção ( $\left.T_{\text {det }}\right)$ é determinado de acordo com a contribuição diária de esgoto calculada para os habitantes ou atividade, conforme Tabela 2 da NBR 7229/1993, utilizou-se 1 dia. Para taxa de acumulação de lodo (K) utilizou-se o valor de 57, que está de acordo com o intervalo de limpeza de um ano e pela faixa de temperatura ambiente considerada maior que $20^{\circ} \mathrm{C}$ (temperatura definida a partir de dados climáticos do site Sistema Meteorológico do Paraná SIMEPAR, 2018), conforme determinado pela Tabela 3 da referida norma.

O filtro anaeróbio e sumidouro foram dimensionados de acordo com a NBR 13969/1997, sendo adotado para preenchimento do filtro brita no 04, especificada pela norma.

Os sistemas de tratamento individuais comparados ao convencional propostos nesse trabalho foram tanque séptico seguido por wetland e fossa biodigestora modelo Embrapa, cujas metodologias seguem descritas abaixo.

a) Tanque séptico seguido de wetland

Conforme descrito anteriormente, o dimensionamento do tanque séptico seguiu NBR 7229/1993.

Para o wetland (W), foi proposto um sistema de fluxo superficial utilizando plantas do tipo Zantedeschia Aethiopica (copo-de-leite) como macrófitas de preenchimento. Os meios filtrantes propostos foram areia grossa e brita $\mathrm{n}-2$. Considerando o volume total do meio 
suporte preenchido por $30 \%$ de areia grossa e $70 \%$ de brita no 2 , com porosidade de 0,31 e 0,51 , respectivamente. Para o dimensionamento, seguiu-se a metodologia proposta por Lemes et al. (2008) e Lisboa (2016).

O cálculo da área ocupada deverá ser calculado pela Equação 1 (Lisboa, 2016):

$$
V=\frac{T D H \times Q}{\alpha}
$$

Sendo:

$\mathrm{V}$ - volume em $\mathrm{m}^{3}$

$Q$ - vazão afluente $\left(m^{3} \cdot d-1\right)$;

TDH - tempo de detenção hidráulica (dias)

$\alpha$-porosidade adimensional

O TDH para o leito com Zantedeschia Aethiopica pode ser adotado como sendo de 1 dia, utilizando areia grossa e brita no 2 (ANDRADE, 2012). Considerou-se o volume do meio suporte constituído por $30 \%$ de areia grossa com porosidade 0,31 e $70 \%$ de brita com porosidade 0,51, encontrou-se uma porosidade média ( $\alpha$ ) de 0,47 (LEMES et al. 2008).

Para cálculo da taxa de aplicação hidráulica (Th) do wetland seguiu-se Lemes et al. 2008, conforme Equação 2.

Sendo:

$$
T h=\frac{Q}{A w i}
$$

$\mathrm{Q}$ - vazão afluente $\left(\mathrm{m}^{3} . \mathrm{d}-1\right)$;

Awi - área de cada wetland construído $\left(\mathrm{m}^{2}\right)$.

Considerando a menor relação de comprimento/largura $=2 / 1$, com a área superficial calculada (Awi), o tamanho dos wetlands construídos será calculado pela Equação (3).

$$
\boldsymbol{A}=\boldsymbol{L} \times \boldsymbol{B}
$$

De acordo com Lisboa (2016), os wetlands construídos da área rural poderão ser impermeabilizados com uma terceira camada de lona plástica ou com argila compactada, com intenção de diminuir os gastos de produção.

b) Fossa biodigestora

A fossa biodigestora (FB) seguiu a proposta de Novaes et al. (2002) que representa o modelo da Empresa Brasileira de Pesquisa Agropecuária - EMBRAPA. Segundo Novaes et al. (2002) o método da Fossa Biodigestora modelo EMBRAPA para uma unidade familiar de até 5 habitantes é formado por três caixas de cimento amianto de $1000 \mathrm{~L}$ cada. Como o modelo segue um padrão, não há dimensionamento, apenas mantém-se as especificações da proposta.

As duas primeiras caixas são ligadas diretamente ao vaso sanitário (portanto recebe apenas águas negras), e a terceira caixa de $1000 \mathrm{~L}$ recebe a coleta do efluente tratado obtendo como produto final o adubo orgânico. As caixas devem estar unidas entre si por canos e ligações de PVC de 4", com curva de 90 extensa dentro das caixas e conexões de inspeção para manutenção. As caixas devem ser enterradas no solo para preservar o isolamento térmico. $O$ procedimento contém dois canos de PVC de $1 \mathrm{~m}$ cada, fixados no meio das duas primeiras 
caixas tampado, mas com pequenos furos laterais, para a saída de gás concentrado. O resíduo final é coletado por meio do registro de esfera de $50 \mathrm{~mm}$ e despejado em caixa coletora para posterior reaproveitamento.

Para o dimensionamento do tanque séptico, filtro anaeróbio, sumidouro e wetland utilizou-se o Excel como ferramenta suporte.

\subsection{Definição dos materiais de construção e orçamento}

A partir do dimensionamento apresentado, as dimensões e proporções para cada unidade do tratamento foram obtidas. Dessa maneira foi feito um levantamento de cada sistema de tratamento com o intuito de apresentar os materiais necessários e orça-los para discussão da viabilidade econômica de dos sistemas propostos. O orçamento foi obtido por meio de pesquisas em 3 depósitos de materiais de construção e 3 empreitas da cidade de Londrina-PR e Sertaneja-PR no período de novembro e dezembro de 2018. A partir desse orçamento, foi realizada uma média para compilação dos dados.

Como comparativo, foi realizado o levantamento de custos utilizando o banco de dados da Tabela de Composições de Preços para Orçamentos - TCPO $13^{\text {a }}$ edição (TCPO, 2008) e do Sistema Nacional de Pesquisa de Custos e índices da Construção Civil - SINAP referência Curitiba (SINAP, 2018). Essa ferramenta foi utilizada para levantamento de custos do sistema TS+FA+S e TS uma vez que não se tem dados disponíveis por meio do TCPO para sistemas wetland e fossa biodigestora.

\section{RESULTADOS}

Os resultados dos dimensionamentos e comparação por viabilidade de área e econômica a partir dos tratamentos propostos estão descritos nos itens subsequentes.

\subsection{Dimensionamento do tratamento convencional (TS+FA+S)}

Considerando uma residência unifamiliar de 5 pessoas com habitação principalmente em finais de semana, o dimensionamento do sistema convencional consta na Tabela 1 e o modelo esquemático é representado pela Figura 1.

Tabela 1 - Dimensões do tratamento convencional

\begin{tabular}{lccc}
\hline & $\begin{array}{c}\text { Tanque } \\
\text { séptico }\end{array}$ & Filtro Anaeróbio & Sumidouro \\
\hline Área $\left(\mathrm{m}^{2}\right)$ & 1,00 & 0,64 & 5,18 \\
Volume $\left(\mathrm{m}^{3}\right)$ & 1,91 & 1,14 & - \\
Largura $(\mathrm{m})$ & 0,75 & - & - \\
Comprimento $(\mathrm{m})$ & 1,50 & - & - \\
Altura $(\mathrm{m})$ & 1,70 & - & 1,10 \\
Diâmetro $(\mathrm{m})$ & - & 0,90 & 1,5 \\
\hline
\end{tabular}


Figura 1 - Modelo esquemático do sistema convencional.

TS

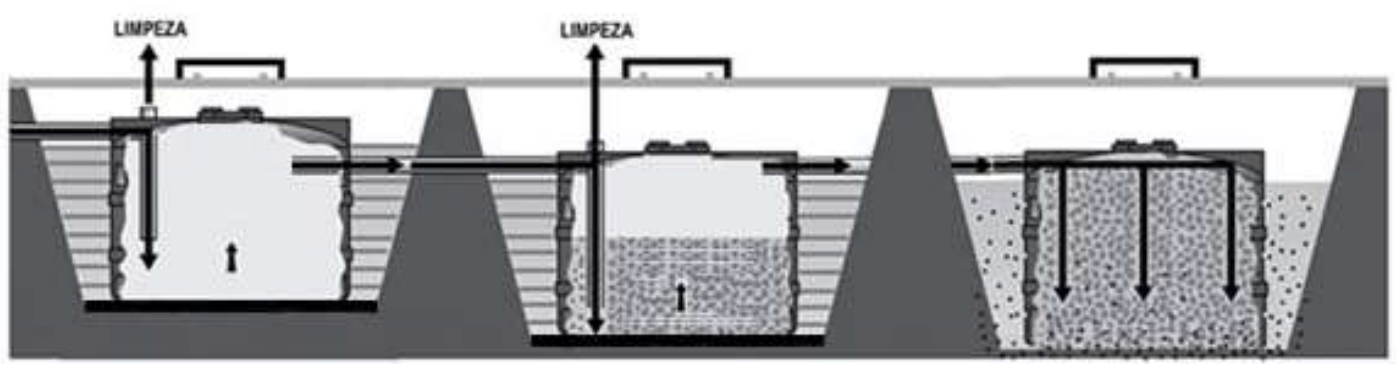

O sistema convencional é muito utilizado por obter eficiência na remoção de resíduos do esgoto bruto. É uma tecnologia viável, possui métodos construtivos e operacionais simplificados. No entanto, requer condições de construção adequadas e maior área de implantação. Em relação ao tratamento, Sperling (2014) e Silva (2014) destacam a eficiência do tratamento anaeróbio quanto à remoção de matéria orgânica e sólidos em suspensão (60 \%), no entanto, não são eficientes quanto à remoção de nutrientes como fósforo e nitrogênio, além da necessidade em assegurar distância ao lençol freático.

\subsection{Dimensionamento dos sistemas alternativos}

A partir das definições citadas na metodologia, a Tabela 2 apresenta o dimensionamento do sistema constituído por TS +W.

Tabela 2 - Dimensões do sistema de tratamento TS + W

\begin{tabular}{lcc}
\hline & $\begin{array}{c}\text { Tanque } \\
\text { séptico }\end{array}$ & Wetland \\
\hline Área $\left(\mathrm{m}^{2}\right)$ & 1,00 & 1,00 \\
Volume $\left(\mathrm{m}^{3}\right)$ & 1,91 & 1,00 \\
Largura $(\mathrm{m})$ & 0,75 & 0,70 \\
Comprimento $(\mathrm{m})$ & 1,50 & 1,40 \\
Altura $(\mathrm{m})$ & 1,70 & 1,00 \\
\hline
\end{tabular}

Para taxa de aplicação hidráulica, a partir da Equação 2, obteve-se o valor de 0,15 m.dia ${ }^{-1}$. Na Figura 2 consta o projeto da construção do sistema, bem como seu aspecto paisagístico.

Figura 2 - Modelo Esquemático TS+W de fluxo superficial

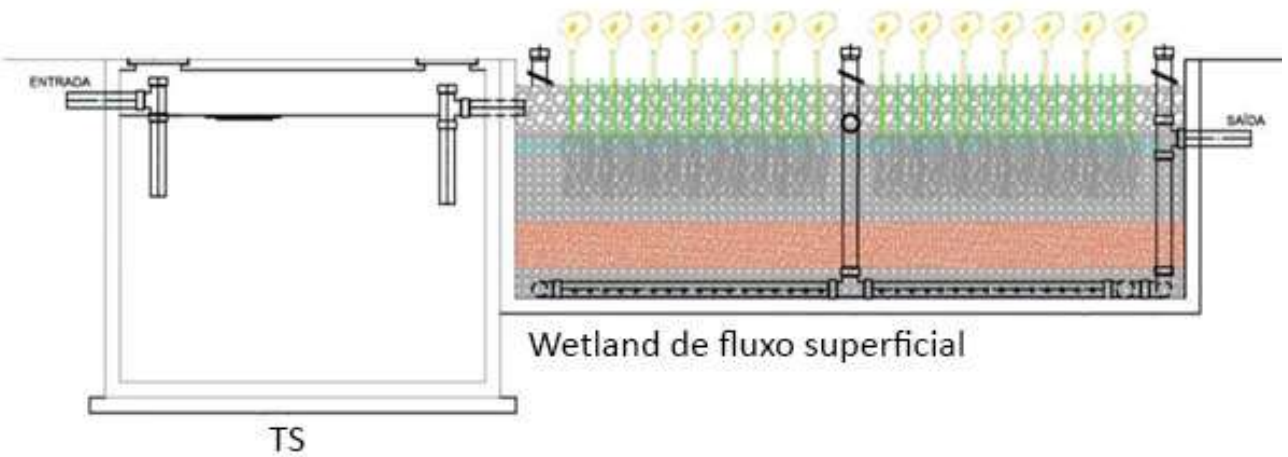


O sistema foi dimensionado para uma unidade familiar e apresenta uma área pouco extensa com tratamento eficiente. De acordo com Sabei e Basseti (2013), wetlands são sistemas eficientes em termos de tratamento (remoção de matéria orgânica, sólidos e nutrientes como fósforo e nitrogênio), minimização de toxicidade além de serem benéficos ao paisagismo. Comparativamente ao modelo convencional de $T S+F A+S$, alguns estudos (SABEl e BASSETI, 2013; LISBOA, 2016) ressaltam que os wetlands são economicamente viáveis e podem agregar valor aos bens e serviços, utilizando de recursos naturais sustentáveis.

Conforme detalhado na metodologia, para a fossa biodigestora modelo EMBRAPA não foi realizado dimensionamento, uma vez que é padronizada pela metodologia de Novaes et al. (2002), sendo constituída por 3 caixas de cimento amianto de 1000 L. A Figura 3 representa o modelo esquemático da fossa biodigestora.

Figura 3 - Modelo esquemático da fossa biodigestora

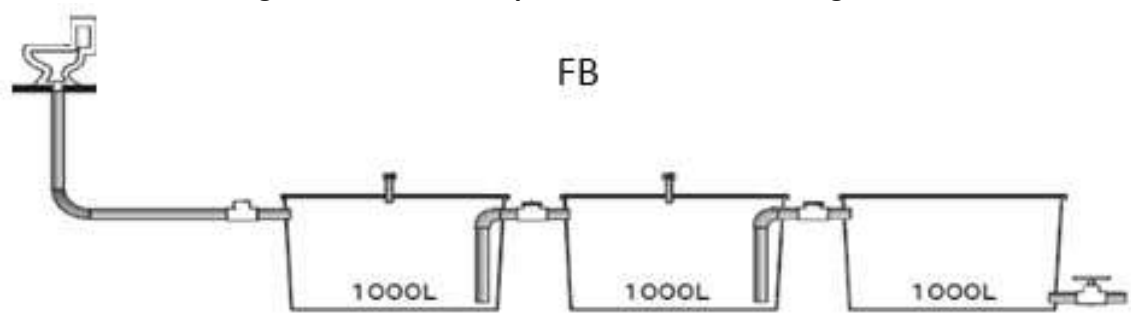

Fonte: Adaptado de Novaes et al. (2002).

A fossa biodigestora em comparação aos sistemas individuais de tratamento de esgoto previstos pela NBR 13969 é previsto como um projeto de custo-benefício vantajoso. A simplicidade de implantação e manutenção além da produção de biofertilizante em curto espaço de tempo (1 mês) a torna interessante para implantação em locais rurais (LEONEL et al. 2013). No entanto, segundo o levantamento realizado por Sabei e Basseti (2013), as desvantagens desse sistema estão relacionadas a restrição quanto à alimentação, uma vez que restringe o tratamento apenas para águas residuárias consideradas negras (oriundas de vasos sanitários), e baixa eficiência na remoção de nutrientes e patógenos.

Dessa forma, os dois sistemas de tratamento propostos nesse trabalho (TS+W e FB), bem como o sistema convencional ( $T S+F A+S)$ possibilitam a minimização de impactos ambientais e sanitários em comparação à fossa rudimentar. Em relação ao dimensionamento, o sistema composto por TS $+\mathrm{W}$ apresentou menor área construída.

\subsection{Viabilidade Econômica}

Para avaliar a melhor aplicação dos três sistemas de tratamento expostos nesse trabalho, a análise de viabilidade econômica, por meio de levantamento de custos foi realizada. Não houve diferenças significativas entre as lojas e empreitas pesquisadas, e, por esse motivo, foram considerados valores médios. Os Quadros 1,2 e 3 representam os valores médios obtidos a partir dos orçamentos de TS+FA+S, TS+W e FB, respectivamente. 
Quadro 1 - Orçamento do sistema TS + FA + S.

\begin{tabular}{|c|c|c|c|c|}
\hline \multicolumn{5}{|c|}{ TANQUE SÉPTICO + FILTRO ANAERÓBIO + SUMIDOURO } \\
\hline \multicolumn{5}{|c|}{ Tanque Séptico } \\
\hline Materiais & Quantidade & Unid. & Valor unit. & Valor total \\
\hline Mão-de-obra & 1 & $\mathrm{~m}^{2}$ & $\mathrm{R} \$ 300,00$ & 300,00 \\
\hline Tijolos 6 furos & 200 & unidade & $\mathrm{R} \$ \quad 0,59$ & 118,00 \\
\hline Areia & 1 & $\mathrm{~m}^{3}$ & $\mathrm{R} \$ 102,00$ & 70,00 \\
\hline Pedra brita & $1 / 2$ & $\mathrm{~m}^{3}$ & $\mathrm{R} \$ 75,00$ & 37,50 \\
\hline Cimento $50 \mathrm{~kg}$ & 2 & saco & $\mathrm{R} \$ 21,40$ & 42,80 \\
\hline Cal Hidratada $20 \mathrm{~kg}$ & $1 / 2$ & saco & $\mathrm{R} \$ 13,29$ & 6,65 \\
\hline Tubo PVC 100 mm 6m & 2 & unidade & $\mathrm{R} \$ 38,90$ & 77,80 \\
\hline Curva PVC $90^{\circ} 100 \mathrm{~mm}$ & 2 & unidade & $\mathrm{R} \$ 11,29$ & 22,58 \\
\hline \multirow[t]{2}{*}{ Laje pré-moldada } & 2,5 & $\mathrm{~m}^{2}$ & $\mathrm{R} \$ \quad 45,00$ & 112,50 \\
\hline & & & TOTAL & 787,83 \\
\hline \multicolumn{5}{|c|}{ Filtro Anaeróbio } \\
\hline Materiais & Quantidade & Unid. & Valor unit. & Valor total \\
\hline Mão-de-obra & 1 & $\mathrm{~m}^{2}$ & $\mathrm{R} \$ 300,00$ & $\mathrm{R} \$ 300,00$ \\
\hline Tijolos 6 furos & 120 & unidade & $\mathrm{R} \$ \quad 0,59$ & 70,80 \\
\hline Areia & 1 & $\mathrm{~m}^{3}$ & $\mathrm{R} \$ 102,00$ & 102,00 \\
\hline Pedra brita no 4 & 0,5 & $\mathrm{~m}^{3}$ & $\mathrm{R} \$ 105,00$ & 52,50 \\
\hline Cimento 50 kg & 1 & saco & $\mathrm{R} \$ 21,40$ & 21,40 \\
\hline Cal Hidratada 20 kg & $1 / 2$ & saco & $\mathrm{R} \$ 13,29$ & 6,65 \\
\hline Tubo PVC 100 mm 6m & 1 & unidade & $\mathrm{R} \$ 38,90$ & 38,90 \\
\hline Curva PVC $90^{\circ} 100 \mathrm{~mm}$ & 2 & unidade & $\mathrm{R} \$ 11,29$ & 22,58 \\
\hline \multirow[t]{2}{*}{ Laje pré-moldada } & \multirow[t]{2}{*}{2} & $\mathrm{~m}^{2}$ & $\mathrm{R} \$ 45,00$ & 90,00 \\
\hline & & & TOTAL & 704,83 \\
\hline \multicolumn{5}{|c|}{ Sumidouro } \\
\hline Materiais & Quantidade & Unid. & Valor unit. & Valor total \\
\hline Tijolos 6 furos & 750 & unidade & $\mathrm{R} \$ \quad 0,59$ & 442,50 \\
\hline Areia & 2 & $\mathrm{~m}^{3}$ & $\mathrm{R} \$ 102,00$ & 204,00 \\
\hline Pedra brita no 2 & 0,34 & $\mathrm{~m}^{3}$ & $\mathrm{R} \$ \mathbf{7 5}, 00$ & 25,50 \\
\hline Cimento 50 kg & 1 & saco & $\mathrm{R} \$ 21,40$ & 21,40 \\
\hline Cal Hidratada 20 kg & 1 & saco & $\mathrm{R} \$ 13,29$ & 13,29 \\
\hline Tubo PVC 100 mm 6m & 1 & unidade & $\mathrm{R} \$ 38,90$ & 38,90 \\
\hline Curva PVC $90^{\circ} 100 \mathrm{~mm}$ & 1 & unidade & $\mathrm{R} \$ 11,29$ & 11,29 \\
\hline Laje pré-moldada & 0,25 & $\mathrm{~m}^{2}$ & $\mathrm{R} \$ 45,00$ & 11,25 \\
\hline & & & & 768,13 \\
\hline & O CONJUNTO & $=A+S$ & & $2.260,78$ \\
\hline
\end{tabular}


Quadro 2 - Orçamento do sistema TS + W

\begin{tabular}{|c|c|c|c|c|}
\hline \multicolumn{5}{|c|}{ TANQUE SÉPTICO + WETLAND } \\
\hline Materiais (TS + WT) & Quant. & Unidade & Valor unit. & Valor total \\
\hline Areia média & 1,5 & $\mathrm{~m}^{3}$ & $\mathrm{R} \$ 102,00$ & $\mathrm{R} \$ \quad 153,00$ \\
\hline Brita $n=1$ & 0,75 & $\mathrm{~m}^{3}$ & $\mathrm{R} \$ 75,00$ & $\mathrm{R} \$ \quad 56,25$ \\
\hline Cimento & 3 & sc $50 \mathrm{~kg}$ & $\mathrm{R} \$ 21,40$ & $\mathrm{R} \$ \quad 64,20$ \\
\hline Cal & 1,5 & sc $20 \mathrm{~kg}$ & $\mathrm{R} \$ 13,29$ & $\mathrm{R} \$ \quad 19,94$ \\
\hline Tijolo & 560 & unid. & $\mathrm{R} \$ \quad 0,59$ & $\mathrm{R} \$ \quad 330,40$ \\
\hline Tubo PVC $100 \mathrm{~mm}(6 \mathrm{~m})$ & 2 & unid. & $\mathrm{R} \$ 38,90$ & $\mathrm{R} \$ \quad 77,80$ \\
\hline Curva PVC $90^{\circ} 100 \mathrm{~mm}$ & 1 & unid. & $\mathrm{R} \$ 11,29$ & $\mathrm{R} \$ \quad 11,29$ \\
\hline Cruzeta PVC $100 \mathrm{~mm}$ & 1 & unid. & $\mathrm{R} \$ 89,00$ & $\mathrm{R} \$ \quad 89,00$ \\
\hline T esgoto $100 \mathrm{~mm}$ & 4 & unid. & $\mathrm{R} \$ 14,58$ & $\mathrm{R} \$ \quad 58,32$ \\
\hline Joelho $90^{\circ} 100 \mathrm{~mm}$ & 3 & unid. & $\mathrm{R} \$ \quad 6,58$ & $\mathrm{R} \$ \quad 19,74$ \\
\hline Joelho $45^{\circ} 100 \mathrm{~mm}$ & 2 & unid. & $\mathrm{R} \$ \quad 5,98$ & $\mathrm{R} \$ \quad 11,96$ \\
\hline Terminal de ventilação 100 mm & 2 & unid. & $\mathrm{R} \$ 24,90$ & $\mathrm{R} \$ \quad 49,80$ \\
\hline Brita $n=2$ & 5,5 & $\mathrm{~m}^{3}$ & $\mathrm{R} \$ 65,00$ & $\mathrm{R} \$ \quad 357,50$ \\
\hline Areia grossa (meio filtrante) & 3,5 & $\mathrm{~m}^{3}$ & $\mathrm{R} \$ 90,00$ & $\mathrm{R} \$ \quad 315,00$ \\
\hline Vegetação & - & unid. & - & - \\
\hline \multirow[t]{2}{*}{ Mão-de-obra } & 2 & $\mathrm{~m}^{2}$ & $R \$ 300,00$ & $\mathrm{R} \$ \quad 600,00$ \\
\hline & & & \multicolumn{2}{|c|}{ TOTAL } \\
\hline
\end{tabular}


Quadro 3 - Orçamento da Fossa Biodigestora

\begin{tabular}{|c|c|c|c|c|c|}
\hline \multicolumn{6}{|c|}{ FOSSA BIODIGESTORA } \\
\hline Materiais & Quant. & Unidade & Valor unit. & Valor tc & tal \\
\hline Caixa amianto $1000 \mathrm{~L}$ & 3 & unid. & $\mathrm{R} \$ 297,48$ & $\mathrm{R} \$$ & 892,44 \\
\hline Tubo PVC $100 \mathrm{~mm}(6 \mathrm{~m})$ & 1 & unid. & $\mathrm{R} \$ 38,90$ & $\mathrm{R} \$$ & 38,90 \\
\hline Válvula de retenção de PVC 100 mm & 1 & unid. & $\mathrm{R} \$ 79,90$ & $\mathrm{R} \$$ & 79,90 \\
\hline Curva PVC $90 \cong 100 \mathrm{~mm}$ & 2 & unid. & $\mathrm{R} \$ 11,29$ & $\mathrm{R} \$$ & 22,58 \\
\hline Luva de PVC $100 \mathrm{~mm}$ & 3 & unid. & $\mathrm{R} \$ \quad 5,69$ & $\mathrm{R} \$$ & 17,07 \\
\hline Tê de inspeção PVC 100 mm & 2 & unid. & $\mathrm{R} \$ 38,90$ & $\mathrm{R} \$$ & 77,80 \\
\hline O'ring $100 \mathrm{~mm}$ (anel de vedação) & 10 & unid. & $\mathrm{R} \$ \quad 0,69$ & $\mathrm{R} \$$ & 6,90 \\
\hline Tubo PVC soldável $25 \mathrm{~mm}$ & 2 & $\mathrm{~m}$ & $\mathrm{R} \$ 10,92$ & $\mathrm{R} \$$ & 21,84 \\
\hline Tubo PVC soldável $50 \mathrm{~mm}$ & 2 & $\mathrm{~m}$ & $\mathrm{R} \$ 25,90$ & $\mathrm{R} \$$ & 51,80 \\
\hline Cap PVC soldável 25 mm & 2 & unid. & $\mathrm{R} \$ \quad 0,99$ & $\mathrm{R} \$$ & 1,98 \\
\hline Flange PVC soldável $25 \mathrm{~mm}$ & 1 & unid. & $\mathrm{R} \$ \quad 9,50$ & $\mathrm{R} \$$ & 9,50 \\
\hline Flange PVC soldável $50 \mathrm{~mm}$ & 1 & unid. & $\mathrm{R} \$ 20,40$ & $\mathrm{R} \$$ & 20,40 \\
\hline Registro de esfera PVC $50 \mathrm{~mm}$ & 1 & unid. & $\mathrm{R} \$ 36,90$ & $\mathrm{R} \$$ & 36,90 \\
\hline Cola de silicone $280 \mathrm{~g}$ & 2 & tubo & $\mathrm{R} \$ 22,90$ & $\mathrm{R} \$$ & 45,80 \\
\hline Borracha de vedação $15 \times 15$ mm & 25 & $\mathrm{~m}$ & $\mathrm{R} \$ \quad 0,78$ & $\mathrm{R} \$$ & 19,50 \\
\hline Pasta lubrificante $\mathrm{p} / \mathrm{PVC}$ rígido & 1 & tubo & $\mathrm{R} \$ 23,31$ & $\mathrm{R} \$$ & 23,31 \\
\hline Adesivo PVC $100 \mathrm{~g}$ & 1 & tubo & $\mathrm{R} \$ \quad 4,13$ & $\mathrm{R} \$$ & 4,13 \\
\hline Neutrol (impermeabilizante asfáltico) & 1 & litro & $\mathrm{R} \$ 22,90$ & $\mathrm{R} \$$ & 22,90 \\
\hline Mão-de-obra & 3 & $\mathrm{~m}^{2}$ & $R \$ 300,00$ & $\mathrm{R} \$$ & 900,00 \\
\hline & & & $\bar{T}$ & $\mathrm{R} \$ 2.29$ & 3,65 \\
\hline
\end{tabular}

Por meio dos quadros apresentados verifica-se que a fossa biodigestora tornou-se o tratamento mais caro com uma diferença de aproximadamente 80,00 reais em relação tratamento por tanque séptico seguido de wetland e de 33,00 comparado ao TS+FA+S. Comparativamente aos três métodos apresentados no artigo, o sistema por wetland foi considerado como o mais viável devido ao menor custo, apesar da pequena diferença.

É importante destacar que o maior custo representado pelo orçamento da fossa biodigestora modelo EMBRAPA está relacionado ao custo de mão-de-obra, uma vez que se manteve R\$ 300,00 por $\mathrm{m}^{2}$. No entanto esse custo pode ser reduzido em outras regiões, dada a simplicidade de implantação e operação do sistema.

Como forma comparativa, o levantamento de custos por meio da TCPO/SINAP apresentou valores reduzidos. O custo total do sistema TS+FA+S foi de $R \$ 2.003,27$, enquanto para 0 sistema de tratamento TS $+W$, em que se considerou a tabela TCPO apenas para o tanque séptico, obteve-se o valor total de $\mathrm{R} \$ 1.979,18$. O levantamento de custos pela TCPO representou uma economia de $R \$ 257,51$ e $R \$ 235,02$ em relação ao levantamento por meio de lojas e empreitas para o TS+FA+S e TS+W, respectivamente. As diferenças de valores representadas entre o levantamento em lojas e empreitas e via TCPO/SINAP são justificadas 
pelas variações regionais e de mercado, mas não implicaram em resultados divergentes quanto ao sistema mais econômico, que foi o TS+W.

Dessa forma, o tratamento por TS+W, destaca-se pelo menor custo e menor área construída, com viabilidade econômica e de implantação. Entre as vantagens do wetland, Sabei e Basseti (2013) e Lisboa (2016) ressaltam a remoção de nutrientes, cujo sistema TS+FA+S não proporciona, além do potencial paisagístico, que possibilita adaptação à vários tipos de espaços.

\section{CONCLUSÃO}

Foram propostos sistemas alternativos de tratamento individual para áreas rurais. Os projetos propostos (TS+W e FB) foram considerados vantajosos quanto ao tratamento, implantação e operação em comparação ao sistema convencionalmente aplicado (TS+FA+S).

Concluiu-se que o tratamento proporcionado pelo sistema wetland está relacionado a uma pequena necessidade de área e baixos custos de implantação. A área total do sistema de tanque séptico seguido de wetland foi de $2 \mathrm{~m}^{2}$ e seu custo foi de $R \$ 2.214,20$, considerado menor e menos custoso comparativamente ao sistema convencional (TS+FA+S $=R \$ 2.260,78) \mathrm{e}$ à fossa biodigestora ( $\mathrm{R} \$ 2.293,65)$. Dessa forma, o sistema wetland apresenta-se como uma alternativa viável para o tratamento de esgoto doméstico em regiões com dificuldade de implantação de rede coletora de esgoto.

Ressalta-se, portanto, a necessidade e a importância em se projetar soluções individuais que minimizem os impactos ambientais e sanitários em áreas rurais, e que sejam economicamente viáveis e sustentáveis.

\section{REFERENCIAS}

ANDRADE, Helisson Henrique Borsato de. Avaliação do Desempenho de Sistemas de Zona de Raízes (Wetlands Construídas) em Escala Piloto Aplicados ao Tratamento de Efluente Sintético. 2012. 87f. Dissertação de Mestrado, Engenharia Civil - Universidade Tecnológica Federal do Paraná, Curitiba, 2012.

ASSOCIAÇÃO BRASILEIRA DE NORMAS TÉCNICAS. NBR 7229/1993: Projeto, construção e operação de sistemas de tanques sépticos. Rio de Janeiro, 1993.

ASSOCIAÇÃO BRASILEIRA DE NORMAS TÉCNICAS. NBR 13969/1997: Tanques sépticos - Unidades de tratamento complementar e disposição final dos efluentes líquidos - Projeto, construção e operação. Rio de Janeiro, 1997.

GONÇALVES, Morgana; KUMMER; Larissa; PATEL, André; SOUZA, Daiane; FROSI, Douglas; FURIGO, Maurício; SAVEGNAGO, Leoberto; TONIAZZO, Fernando. Educação ambiental em saneamento rural. Revista Engenharia Ambiental, Espírito Santo do Pinhal, v. 10, n. 2, p. 255-260, mar /abr 2013.

GONTIJO, H. M. Sustentabilidade econômica no sistema de água e esgoto na comunidade rural de Amadeu Lacerda em Divinópolis/MG, Dissertação de mestrado, Universidade Federal de Lavras,LavrasMG, 90p., 2016. 
IBGE - Instituto Brasileiro de Geografia e Estatística. Pesquisa Nacional por Amostra de Domicílios. Banco da Dados Agregados (PNAD), 2011.

LEMES, J. L. V. B.; SHIRMER, W.N.; CALDEIRA, M.V.W.; KAICK, T.V.; ABEL, O.; BARBARA, R.R; Tratamento de esgoto por meio de zona de raízes em comunidade rural. Rev. Acad., Ciênc. Agrár. Ambient., Curitiba, v. 6, n. 2, p. 169-179, abr./jun. 2008.

NOVAES, A.P.; SIMOES, M.L.; NETO, L.M.; CRUVINEL, P.E.; SANTANA, A.; NOVONUTY, E.H.; SANTIAGO, G.; NOGUEIRA, A.R.A.; Utilização de uma Fossa Séptica Biodigestora para Melhoria do Saneamento Rural e Desenvolvimento da Agricultura Orgânica. São Carlos: Embrapa, 2002.

LEONAL, L. F.; MARTELLI, L.F.A.; SILVA, W.T.L.; Avaliação do efluente de fossa séptica biodigestora e jardim filtrante, Anais de Eventos, III Symposium on agricultural and agroindustrial waste management, São Pedro - SP, Brasil, 2013.

LISBOA, S. M. F. Soluções Alternativas de Saneamento Básico para Comunidades Rurais. 2016. 130 f. TCC (Graduação) - Curso de Engenharia Sanitária e Ambiental, Universidade Federal do Recôncavo da Bahia Ufrb, Cruz das Almas, 2016.

PERES, Leandro José Simoni; HUSSAR, Gilberto José; BELI, Euzebio. Eficiência do tratamento de esgoto doméstico de comunidades rurais por meio de fossa séptica biodigestora. Revista Engenharia Ambiental, Espírito Santo do Pinhal-SP, v. 7, n. 1, p. 020-036, jan. /mar, 2010.

PLANO NACIONAL DE SANEAMENTO BÁSICO -PLANSAB, Panorama do saneamento básico no Brasil, Ministério das cidades, Secretaria Nacional de Saneamento Ambiental, p. 172, 2013.

SABEI, T.R.; BASSETI, F.J.; Alternativas ecoeficientes para tratamento de efluentes em comunidades rurais, Fórum Ambiental da Alta Paulista, v.9; n.11; 2013.

SILVA, D.F.; MOREJON, C.F.M.; LESS, F.R.; Prospecção do panorama do saneamento rural e urbano no Brasil, Revista eletrônica do Mestrado em Educação Ambiental, v. especial, p. 254 -257, 2014.

SISTEMA METEOROLÓGICO DO PARANÁ -SIMEPAR - site: www.simepar.br/ (Acesso: 2018).

SISTEMA NACIONAL DE PESQUISA DE CUSTOS E ÍNDICES DA CONSTRUÇÃO CIVIL -SINAP, Caixa Econômica Federal, 2018 site: http://www.caixa.gov.br/poder-publico/apoio-poderpublico/sinapi/Paginas/default.aspx (Acesso:2018).

SPERLING, M. von. Introdução à qualidade das águas e ao tratamento de esgotos. 4. ed. Belo Horizonte: UFMG, 2014.

TCPO - Tabela de composição de Preço para Orçamento. São Paulo. PINI, 2008. 630 p.13를 ed. 